\title{
Assessment of the BWB aircraft for military transport
}

\author{
Sajal Kissoon \\ Francesco Saverio Mastropierro \\ Devaiah K. Nalianda \\ Andrew Rolt \\ Bobby Sethi \\ Propulsion Engineering Centre, \\ School of Aerospace, Transport and Manufacturing \\ Cranfield University \\ Bedford, UK
}

\begin{abstract}
Purpose - The growth in air mobility, rising fuel prices and ambitious targets in emission reduction are some of the driving factors behind research towards more efficient aircraft. The purpose of this paper is to assess the application of a blended wing body (BWB) aircraft configuration with turbo-electric distributed propulsion in the military sector and to highlight the potential benefits that could be achieved for long-range and heavy payload applications.

Design/methodology/approach - Mission performance has been simulated using a point-mass approach and an engine performance code (TURBOMATCH) for the propulsion system. Payload-range charts were created to compare the performance of a BWB aircraft with various different fuels against the existing Boeing 777-200LR as a baseline.

Findings - When using kerosene, an increase in payload of 42 per cent was achieved but the use of liquefied natural gas enabled a 50 per cent payload increase over a design range of 7,500 NM. When liquid hydrogen (LH2) is used, the range may be limited to about 3,000 NM by the volume available for this low-density fuel, but the payload at this range could be increased by 137 per cent to $127,000 \mathrm{~kg}$.

Originality/value - The results presented to estimate the extent to which the efficiency of military operations could be improved by making fewer trips to transport high-density and irregular cargo items and indicate how well the proposed alternatives would compare with present military aircraft. There are no existing NATO aircraft with such extended payload and range capacities. This paper, therefore, explores the potential of BWB aircraft with turbo-electric distributed propulsion as effective military transports.
\end{abstract}

Keywords Blended wing body, Liquefied natural gas, Liquefied hydrogen, Novel aircraft, Hybrid-electric aero propulsion systems for military applications, Liquid hydrogen

Paper type Research paper

\section{Nomenclature}

$\begin{array}{ll}\text { KE } & =\text { Kerosene; } \\ \text { LNG } & =\text { Liquefied Natural Gas; } \\ \mathrm{LH}_{2} & =\text { Liquid Hydrogen; } \\ \mathrm{NM} & =\text { Nautical Mile; } \\ \text { OEW } & =\text { Operating Empty Weight; } \\ \text { MRTT } & =\text { Multi-Role Tanker Transport; } \\ \text { MTOW } & =\text { Maximum Take-Off Weight; and } \\ \text { ETRW } & =\text { Energy to Revenue Work Ratio. }\end{array}$

\section{Introduction}

Commercial aviation is a continuously growing market as increasing affordability has enabled the number of passengers travelling by air to more than double over the past 20 years. This growth in mobility is expected to continue and nearly double again to 7.8 billion trips per annum by 2036 (IATA, 2017).

This raises a serious concern. According to the European Commission in 2017, growth in aviation has led to it accounting for about 3 per cent of the EU's total greenhouse gas emissions and more than 2 per cent of global emissions. With the increasing number of people travelling by air, it is projected that by 2020 , emissions from global aviation will be 70 per cent higher and by 2050 could grow by $300-700$ per 
cent, relative to 2005, if actions are not taken (European Commission, 2017). Aviation within the military sector is also faced with the need to curb emissions while maintaining the levels of performance required to ensure national security. For example, a study by Waitz et al. (2005), foresaw that despite the small and decreasing fraction of fossil fuel consumed by military aircraft in the USA, the tightening of regulations on community noise and air quality was expected to become a major cause for concern. Additionally, there is a general trend to invest in more versatile aircraft, which can serve multiple roles. These include rescue missions, serving as aerial medical platforms, mid-air refuelling, tactical/strategic and operational airlift and aerial reconnaissance. Heavy lifters are used to transport personnel and military equipment. In the USA, cargo aircraft account for 16 per cent of the Air Force's total number of operations (CBO, 2016).

This paper aims at presenting the results and assessing the applicability of a new aircraft configuration in military applications based on the research undertaken on the NASA N3-X aircraft, which was designed primarily for civil aviation, and was assessed on its lower environmental footprint and economic/investment viability (Felder et al., 2009). The suitability and possible applications of a blended wing body (BWB) aircraft with a boundary layer ingesting (BLI) turboelectric distributed propulsion system is considered here for long-range and heavy-payload applications in a military environment.

Some differences arise between the requirements of a civil cargo aircraft, which focusses more on the speed and efficient delivery of containers to well-equipped terminals, and a military cargo aircraft that relies more on its ability to carry items of large volume and irregular size and/or high density, have a quick response to emergencies and unload at poorlyequipped airfields.

For military application, the BWB configuration is particularly attractive for cargo, tanker and arsenal aircraft missions and is proposed by Funk et al. (2006) to help alleviate governmental spending and potentially replace the C-5 fleet and add to the C-17 fleet. The interest in this particular configuration lies in its ability to provide more fuel-efficient transport of cargo and load particularly large and irregular pellets so as to reduce the number of flights, which would be required by conventional airplane configurations. Additionally, because of its wide airframe, the possibility of refuelling multiple aircraft at the same time occurs. However, because of the large span of the airframe, folding tip mechanisms may need to be considered. This is highlighted within the EC Framework 6 project new aircraft concept research, which was undertaken to integrate and validate technologies that enable new aircraft concepts to be assessed.

Through the silent aircraft initiative, 35 researchers from Cambridge and MIT investigated the feasibility of an ultra-low noise, fuel efficient BWB. They came up with the SAX-40, which integrates novel and advanced noise minimising aircraft systems with aerodynamic shaping of the airframe centre-body. It includes BLI engines, which aimed at reducing airframe drag by re-energising the boundary layer of the airframe. Later on, NASA developed the N3-X within the subsonic fixed wing (SFW) project, which proved that this BWB aircraft would be able to provide at least a 60 per cent reduction in total energy consumption as compared to the best in class current generation aircraft (Felder et al., 2011). A representation of NASA's N3-X configuration is depicted in Figure 1.

A BWB aircraft with distributed propulsion systems is considered to be one of the most promising disruptive technologies under research. The innovative aircraft design offers high aerodynamic and propulsive efficiency savings and better payload capacity than a conventional technology aircraft with similar take-off weight (Liebeck, 2004).

This paper assesses the application of the N3-X configuration to military missions and its potential for improving fuel consumption while reducing emissions. This is achieved through a synergistic mix of technological improvements to the airframe and propulsion system, and though the use of different fuels for the propulsion system, namely, kerosene, hydrogen and liquefied natural gas (LNG).

\section{State of the art}

The Antonov An-225 (enlargement of the Antonov An-124) is the largest aircraft used to transport heavy payloads. It can carry a maximum of $250,000 \mathrm{~kg}$ over a range of 2,444 $\mathrm{NM}$ and has a ferry range of $8,363 \mathrm{NM}$ (Airliners). It was originally built to carry the Buran spaceplane and was later used to carry military supplies including a record load of four main battle tanks. Only one An-225 was completed, but it remains in operation.

The USA air force cargo aircraft fleet is mainly comprised of the C-130 Hercules (which also make up 25 per cent of all cargo planes globally). These aircraft can carry a maximum payload of 16,330 kg over a range of 2,049 NM and have a ferry range of 5,200 NM (GlobalSecurity, 2011). Although its payload is only 13 per cent that of the An-225, the aircraft is valued for its optimal combination of low acquisition cost and useful cargo space for medium to large payload applications. The continuing popularity of the C-130 demonstrates the ongoing demand for military airlifters. This is further reinforced by the introduction of the A $400 \mathrm{M}$ by Airbus. This multi-role heavy-lift aircraft, although of similar wingspan to the C-130, has a maximum payload of $37,000 \mathrm{~kg}$ and is able to achieve a maximum range of 4,800 NM (Airbus).

As a larger-payload aircraft, the C-17 Globemaster is not only highly valued for strategic airlift requirements but is also versatile in terms of its capability of performing short field

Figure 1 NASA's N3-X aircraft

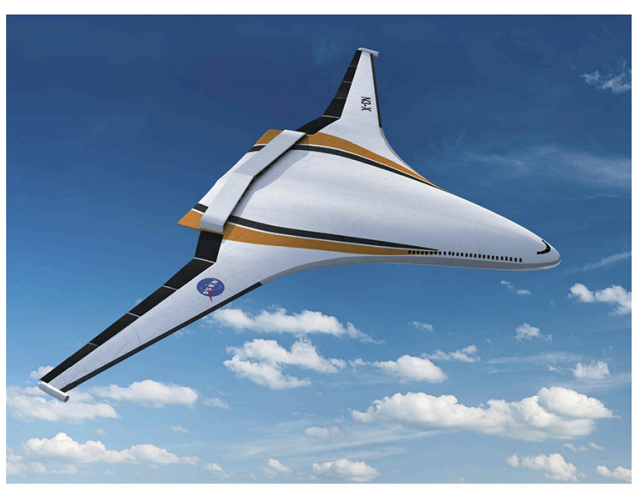

Source: Image Credit: NASA 
landings at full cargo load and acting in tactical airlift and airdrop missions. It can carry a maximum payload of approximately $77,500 \mathrm{~kg}$. With a payload of $60,000 \mathrm{~kg}$, the C17 has a range of 5,200 NM (FAS, 2000). Like the C-130, it can carry troops and equipment close to the front lines due to its ability to use short runways. The C-5M Galaxy, on the other hand, is used to transport heavy payload to military bases. It achieves a range of 5,500 NM with a payload of $129,000 \mathrm{~kg}$ (FAS, 2000).

In terms of air-to-air refuelling capabilities, the Airbus A330 multi-role tanker transport (MRTT) and the Boeing KC-46 are the current market leaders. With a maximum fuel capacity of $111,000 \mathrm{~kg}$ and additional space for a payload of $45,000 \mathrm{~kg}$, the A330 MRTT is used for special missions such as military transport, refuelling and Airborne Warning and Control System (GlobalSecurity, 2015). However, because this aircraft is derived from the A330, it may prove to be less economical in terms of fuel consumption compared to the newer Airbus models. Comparatively, the $\mathrm{KC}-46 \mathrm{~A}$ has a fuel capacity of $96,300 \mathrm{~kg}$ and a maximum payload of $30,000 \mathrm{~kg}$ (U.S. Air Force, 2019). However, in Poland and South Korea, it failed to secure contracts due to a preference towards the A330 MRTT. Additionally, its delivery to the USA Air Force was heavily delayed due to technical difficulties, which sparked some criticism within the Pentagon and the USA Congress (Charpentreau, 2019). Table I summarises the specifications of modern and popular large transport aircraft and gives a clear understanding as to their payload and range capabilities.

In terms of high payload and range with a low fuel consumption and low noise, the BWB aircraft is proposed in this paper as a competitive candidate for strategic airlifting missions and air-to-air refuelling, which is presented in later sections.

\section{Cargo capacities}

According to Whitford (1999), one of the main requirements for the design of a cost-effective airlifter is to ensure that the cross-sectional area of the cargo deck allows for the regular carriage of irregular equipment and vehicles, including highpriority, low-to-medium density freight. For example, the cargo hold for the C-50 airlifter has a width of $4 \mathrm{~m}$ (height of $3.7 \mathrm{~m}$ and length of $17 \mathrm{~m}$ ), which allows the transport of light vehicles side-by-side.

Mari (2015), found that the N3-X sized BWB aircraft has a total storage volume of approximately $590 \mathrm{~m}^{3}$, which is greater than the Airbus A400M, which has a cargo volume of $270 \mathrm{~m}^{3}$. Though less volume than the C-5 $\left(656 \mathrm{~m}^{3}\right)$ or the AN-225 $\left(1,100 \mathrm{~m}^{3}\right)$, the greater width of the BWB aircraft's cargo hold may allow better use of the space available. Additionally, for shorter flight ranges, the cargo capacity could be increased by reducing the storage space allocated for fuel by installing removable tanks. Figure 2 illustrates potential storage spaces in the N3-X. The deck for passengers could accommodate 300 people, while the storage space allocated for the main cargo (width of $5.9 \mathrm{~m}$, length of $14.3 \mathrm{~m}$ and height of $2 \mathrm{~m}$ ) allows the carriage, for example, of up to nine lightweight Range Rover vehicles. The two side cargo decks each provide an additional $31 \mathrm{~m}^{3}$ of storage space.

\section{Alternative fuels}

The main alternative fuels considered are LNG and $\mathrm{LH}_{2}$, which according to Brewer (1976) are the most "reasonable" alternatives for future aircraft applications. Because of its availability, natural gas is gaining in popularity throughout the world as an alternative fossil fuel and potential biofuel. With approximately 93 per cent of LNG being methane, the lower carbon to hydrogen ratio gives a reduction in carbon emissions. However, using hydrogen would totally eliminate carbon emission in aviation if the production of liquid hydrogen could be carbon-free.

With a specific energy approximately 2.8 times higher than kerosene, a hydrogen fuelled aircraft can have a lower take-off weight for a given payload. Although the operating empty weight of the aircraft will increase because of the additional structural weight of the cryogenic tanks, a reduction in missionlevel energy consumption can be predicted (Goldberg, 2018). This benefit is further enhanced if the range of the aircraft is increased. The major drawback of using liquefied hydrogen is its low density, which even in its liquid state $\left(-253^{\circ} \mathrm{C}\right)$ requires four times as much volume as kerosene. An increase in the volume of the airframe has a negative impact on aerodynamic efficiency, though BWB airframes reduce this downside.

LNG offers a compromise between increased specific energy and increased fuel volume relative to kerosene and $\mathrm{LH}_{2}$. The density of methane is closer to that of kerosene, so a lower volume is required to store the same mass of fuel compared to hydrogen, but the lower heating value (LHV) of methane is also closer to kerosene. When compared to hydrogen, although the volume required is reduced, a greater mass of fuel needs to be stored due to its lower energy content (2.4 times lower than hydrogen). Density is the dominating factor and there is a net reduction in volume compared to liquid hydrogen, and a potential improvement in lift to drag ratio depending on the modifications made to the aircraft.

With the successful flight of the LNG-fuelled Tupolev Tu155 aircraft in 1989, it was demonstrated that it would be

Table I Summary of popular large transport aircraft

\begin{tabular}{lcccc}
\hline & Max payload (kg) & Max fuel capacity (kg) & Max payload range (NM) & Ferry range (NM) \\
\hline Antonov An-225 & 250,000 & 300,000 & 2,444 & 8,363 \\
Lockheed C-130 Hercules & 16,330 & 27,200 & 2,049 & 4,800 \\
Airbus A400M & 37,000 & 50,500 & 2,420 & 4,800 \\
Boeing C-17 Globemaster & 77,500 & 108,000 & 5,500 & 6,230 \\
Lockheed C-5 Galaxy & 129,000 & 155,000 & - & 7,000 \\
Airbus A330MRTT & 100,000 & 111,000 & - & 8,000 \\
Boeing KC-46 & 30,000 & 96,300 & 6,385 \\
\hline
\end{tabular}


Figure 2 Cargo spaces for the N3-X

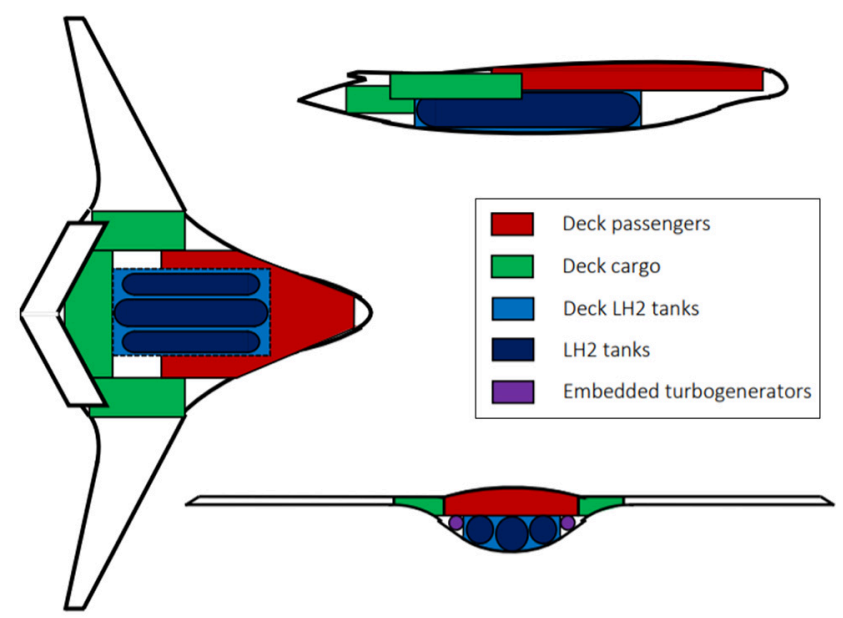

Source: Mari (2015)

feasible within a short timeframe to introduce LNG into aviation. With the added advantage of higher combustion performance (higher stability limits) than kerosene, LNG is a strong candidate for the initial transition from kerosene. Research is currently still ongoing regarding the implementation of hydrogen in aviation. It is expected to take longer than LNG to be introduced to the market (European Commission, 2018).

\section{Methodology}

The approach used is an extension of previous research work conducted on the NASA N3-X aircraft (Felder et al., 2011). One of the main aims was to assess the potential of this novel configuration to achieve NASA's $\mathrm{N}+3$ goal of a 60 per cent reduction in energy use by 2035 compared to the 2005 best in class baseline aircraft (Boeing 777-200LR). Through Technoeconomic and environmental risk assessment, a holistic evaluation was obtained, which integrates the performance of the airframe, the propulsion system, economics, environmental impact, noise, emissions and cost in a modular framework (Ogaji et al., 2007). The assessment was performed on the kerosene and $\mathrm{LH}_{2}$ variants of the aircraft and the results were summarised by Goldberg (2018). The performance assessment was also extended to the LNG variant because of the favourable properties of this fuel, which offers benefits compared to kerosene (higher LHV) and hydrogen (higher density). Because of the study of three different types of fuel used to generate revenue within air transport, a uniform metric was required to assess the energy efficiency of converting the fuel into useful work. The energy to revenue work (ETRW) ratio was found to be the most appropriate metric as it quantifies the energy liberated during a flight with respect to the work done for which a revenue may be earned.

\section{N3-X propulsion system modelling}

The concept consists of a BWB airframe powered by an array of BLI fans as shown in Figure 3. The propulsion system is made up of two embedded turbo-generators, which create enough
Figure 3 N3-X configuration

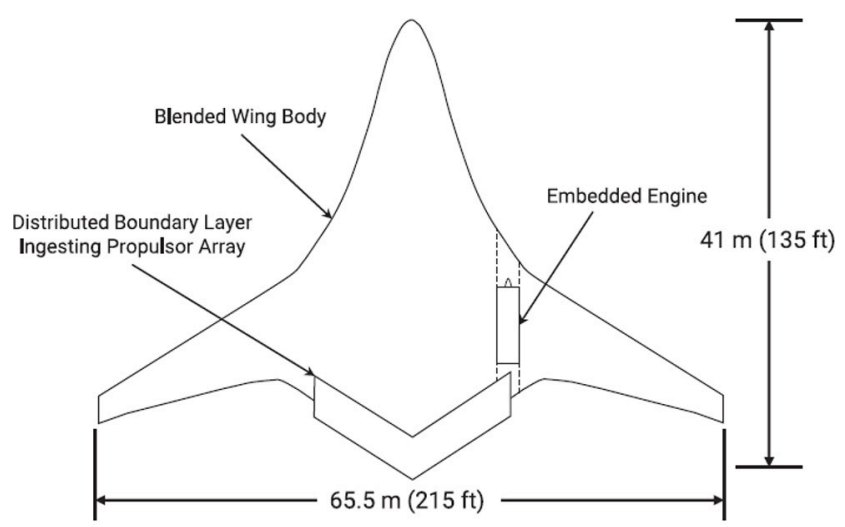

Source: Goldberg (2018)

power to drive 15 BLI propulsors and additionally just enough thrust at design point to overcome their inlet momentum drag. Power is then assumed to be distributed to the propulsion array using superconductors and cryo-cooled transmission systems, which are required to reduce losses and improve efficiency without incurring a significant weight penalty. An efficiency of 99.8 per cent was used to account for transmission losses. The turbo-generators were modelled using TURBOMATCH (Macmillan, 1974), Cranfield's in-house engine performance software. A twin-spool configuration was assumed and the design-point parameters were set according to previous research work by Felder et al. (2011).

The fans are designed at the top of the climb to produce $119 \mathrm{kN}$ of net propulsive force, which is divided equally among the propulsors (Felder et al., 2011). Their performance was predicted using the inlet flow characteristics by averaging the properties of the boundary layer and free stream at the inlet of each propulsor. Hence, using conventional thrust and drag bookkeeping methods, the performance of the array was estimated. Similarly, for the off-design operation, the mass flow at the inlet was varied according to the thrust requirements. Therefore, variable nozzle area was assumed for the propulsor array.

\section{Flight performance model}

The flight performance results were generated using a pointmass approach and an in-house code specifically designed to account for the novel architecture and propulsion system of the N3-X.

The mission performance results are calculated by combining the block fuel burn of several flight phases, namely, taxi, take-off, climb, cruise, descent and landing. Data for each phase result from the interpolation of a pre-defined number of points, at which both aircraft and engine parameters are calculated. Aircraft drag estimation relies on a build-up method, where profile drag is calculated by summing the contribution of each airframe component. Established empirical formulations (Jenkinson et al., 1999) are used; liftinduced drag correction and transonic wave drag are also considered. As blended wing bodies are similar to flying wings, the aerodynamic wing area is assumed equal to the fuselage 
plus the wing planform area. Climb and descend schedules are fixed, and TET is used as engine handle. Energy share factors (ESDU, 1992) are adopted to calculate the correct rates of climb and descent, when changes in velocity and altitude occur simultaneously.

The cruising altitude was taken to be $40,000 \mathrm{ft}$ at a Mach number of 0.84 . The $\mathrm{N} 3-\mathrm{X}$ is estimated to have a higher speed at cruise (Mach 0.84) compared to the $\mathrm{C}-17$, which flies at a Mach number of 0.77. Apart from fuel required for the mission, additional reserve fuel was stored, which includes fuel required for a $200 \mathrm{NM}$ diversion to an alternative airport and an additional 5 per cent of total fuel weight. The dimensions of the airframe model were obtained from the public domain (Moore, 2014) and the weight from previous research work (Goldberg, 2018).

With the Boeing 777-200LR as the baseline aircraft, the performance of the N3-X variants were assessed in terms of their ability to meet NASA's SFW goals (Felder et al., 2011). Because of a higher lift to drag ratio and through the use of boundary layer ingestion within the propulsion system, the BWB is predicted to achieve a better performance in terms of fuel consumption compared to the baseline aircraft.

\section{Results}

A payload range chart was used to assess the differently-fuelled variants of the N3-X and to compare them against the Boeing $777-200 \mathrm{LR}$ with a passenger capacity of over 300 and a design range of 7,500 NM with maximum payload. In 2006, Boeing considered a derivative KC-777 Strategic Tanker in response to the USAF KC-X request for proposals (Tirpak, 2009). The key weight characteristics of the baseline aircraft and the proposed N3-X variants are summarised in Table II.

The maximum take-off weight (MTOW) of the aircraft is made up of the operating empty weight, payload and fuel. The operating empty weight of the N3-X (kerosene version) was obtained from previous research (Felder et al., 2011) as was the MTOW (Goldberg, 2018). Because of the structural capability of the airframe, the MTOW is constant across all variants. This leads to the $\mathrm{LH}_{2}$ variant being underutilised as the sum of its operating empty weight, maximum payload and maximum fuel being less than its MTOW. This is seen in Figure 4 where for the $\mathrm{LH}_{2}$ variant, the points at the maximum payload range and at the maximum fuel range coincide.

The sizing of the tank was performed for the $\mathrm{LH}_{2}$ and LNG variants to take into consideration the additional thickness required to insulate the tanks to prevent excessive boil-off of the fuel and maintain the structural integrity of the tank at the varying tank pressures throughout the flight (Goldberg, 2018). Integrally-insulated tanks were assumed to prevent the deposition of ice on cold surfaces. This increase in tank weight
Figure 4 Payload-range chart at a fixed maximum design payload of $53570 \mathrm{~kg}$

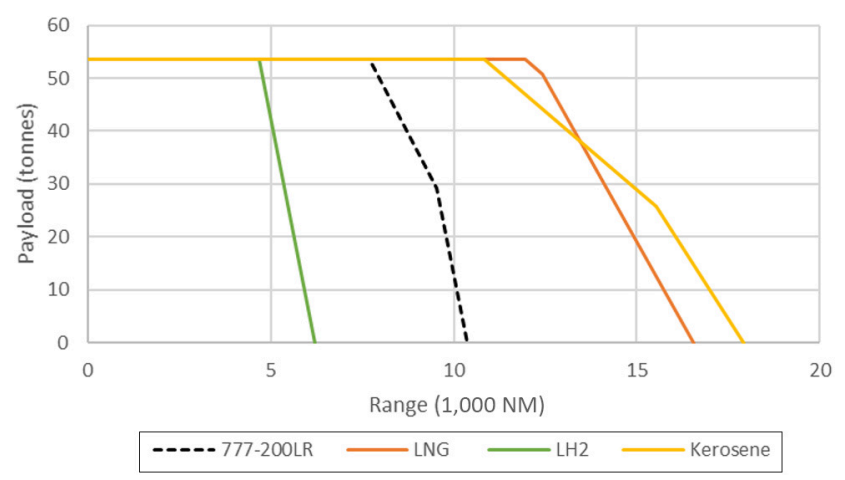

increased operating empty weight for both the $\mathrm{LH}_{2}$ and LNG variants as can be seen in Table II.

Because the volume allocated for fuel storage was assumed to be the same as for the kerosene version, the maximum fuel mass varied for the alternative fuels based on their density and insulation thicknesses. However, higher heating values partially compensated for the lower densities.

With the design payload fixed to $53,570 \mathrm{~kg}$, the payload range chart obtained is shown in Figure 4.

The LNG variant of the N3-X was initially designed to produce the same net propulsive force at the aerodynamic design point as the kerosene-fuelled N3-X model. This was a reasonable assumption given that it was assumed that only the fuel changes and that the two aircraft have similar weight and drag at top of the climb on a MTOW limited mission. Given that the fuel capacity in the LNG version is restricted, it can only carry 85.5 per cent of the energy of the kerosene version. However, due to its lower density, the maximum mass of LNG carried is only 78 per cent of the maximum mass of kerosene. As a consequence, its range at maximum payload exceeds that of the kerosene fuelled N3-X by about 10 per cent, but its maximum ferry range is about 10 per cent less.

The impact on range due to fuel storage capacity is even more severe when considering $\mathrm{LH}_{2}$. In this case, the maximum payload range was reduced by approximately 56 per cent compared with the kerosene version. The fact that there is no difference between the maximum payload range and maximum fuel range means that the aircraft is highly underutilised and that more high density payload can be stored.

Figure 5 shows the extent to which the maximum payload may be increased.

The payloads for LNG and kerosene configurations were increased until the maximum payload range coincided with the design range of the baseline aircraft (777-200LR). With LNG,

Table II Key weight parameters of the various configurations

\begin{tabular}{lcrrr}
\hline & Kerosene & N3-X variants & Baseline \\
& LH & LNG & 777-200LR \\
\hline Maximum take-off weight $(\mathbf{k g})$ & 267,400 & 267,400 & 267,400 \\
Operating empty weight $(\mathbf{k g})$ & 121,290 & 126,770 & 122,600 & 347,450 \\
Maximum payload (kg) & 53,570 & 53,570 & 53,570 & 155,530 \\
Maximum fuel (kg) & 120,284 & 12,910 & 94,100 & 53,570 \\
\hline
\end{tabular}


Figure 5 Payload-range chart for extended payload capacities

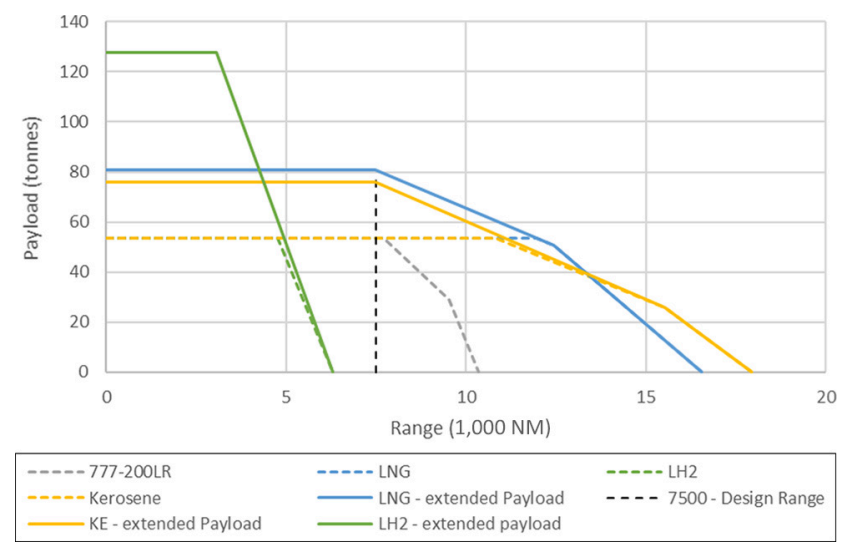

the maximum payload achieved was $80,887 \mathrm{~kg}$, which is about a 50 per cent improvement over the baseline. For the kerosene version, the improvement was approximately 42 per cent.

Considering the hydrogen variant, the sum of the maximum payload, maximum fuel and operating empty weight is lower than the MTOW. If we extend the payload until the MTOW is reached, the maximum payload would be about $127,000 \mathrm{~kg}$, which is approximately 137 per cent more payload than the baseline aircraft. However, the maximum payload range would decrease to about 3,000 NM, which is approximately the distance between London and Toronto and is classified as a short/medium haul flight. The benefit gained from the low density of hydrogen is slightly reduced due to the higher operating empty weight of the aircraft.

The results were also compared against that of the Airbus A $400 \mathrm{M}$, which was built as a tactical airlifter. From Figure 6, the superiority of the N3-X in terms of payload and range is demonstrated. Although the attractiveness of the A400M stems from its flexibility of operations, its payload and range do not match variants of the N3-X. For instance, the hydrogen variant could allow for short field take-off at a much higher payload and for longer range.

The Airbus A330 MRTT is a closer match to the N3-X derivatives in terms of its capabilities. It can take a maximum payload of 45,000 kg (not including fuel in its tanker role) over

\section{Figure 6 Payload-range performance compared to airbus A400M}

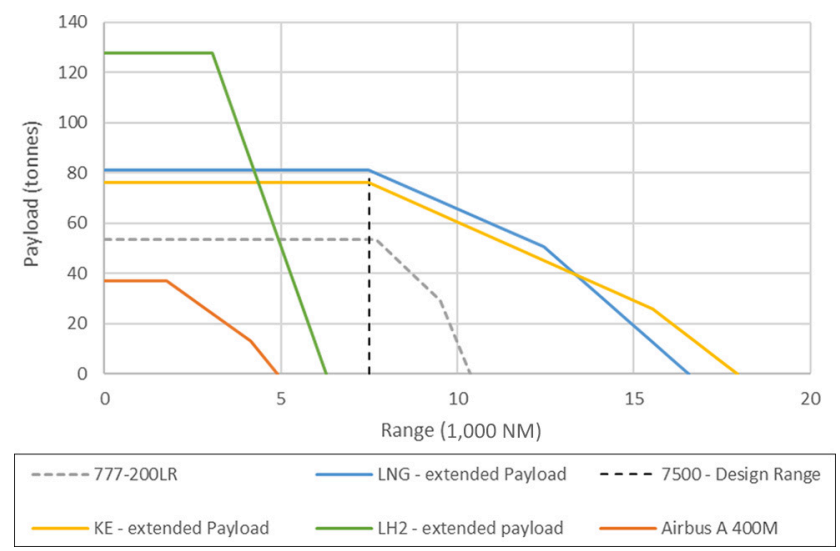

4,000 NM and it has a maximum ferry range of 8,000 NM. The Boeing KC-46A is another slightly-smaller aircraft with similar capabilities.

Because of the different fuels, a separate metric is required to compare the efficiency with which a specific mission is performed. In this scenario, the ETRW ratio is adopted. It is a measure used in the assessment of an aircraft that provides an indication of how efficiently energy is used to generate revenue. This metric is particularly useful when comparing different technologies. Also, it links the energy efficiency with the ability of the aircraft to generate revenue. Hence, using the same metric, different missions can be assessed. A low value indicates high energy efficiency.

$$
E T R W=\frac{m_{f} \cdot L H V}{M_{p l} \cdot g \cdot R}
$$

Where $\mathrm{mf}$ is the mission fuel burn (kg), LHV is the LHV of the fuel $(\mathrm{MJ} / \mathrm{kg}), \mathrm{Mpl}$ is the flight payload $(\mathrm{kg}), \mathrm{g}$ is the acceleration due to gravity $\left(\mathrm{m} / \mathrm{s}^{2}\right)$ and $\mathrm{R}$ is the flight range $(\mathrm{NM})$.

Using the ETRW ratio, the performance of the variants can be assessed on a common ground regardless of the type of fuel used or range achieved. Figure 7 gives a general view of how the "energy efficiency" or ETRW varies with range. The lower the ETRW, the lower is the energy consumption and the higher is the energy efficiency. The baseline aircraft has a much higher ETRW as compared with the N3-X configurations. This is due to the lower lift to drag ratio of the tube and wing configuration, compared with the BWB.

Considering the various $\mathrm{N} 3-\mathrm{X}$ variants, when range is increased from 2,000 NM, the ETRW decreases because of the relatively lower impact of the weight of the fuel used for take-off and climb as range is increased. As range increases, the advantage of using a lighter fuel becomes more apparent. Hydrogen has the lowest increase in ETRW as range increases.

Compared with the kerosene variant, the ETRW of LNG at a given range and payload is approximately 1 per cent lower, while for hydrogen variant; the index is 8 per cent lower. This is mainly because of the greater similarity of LNG to kerosene in terms of LHV as compared with hydrogen.

The energy saving potential of the N3-X variants was assessed against the baseline aircraft and shown to give

Figure 7 Variation of the ETRW index against range for the N3-X variants (Payload: $53570 \mathrm{~kg}$ )

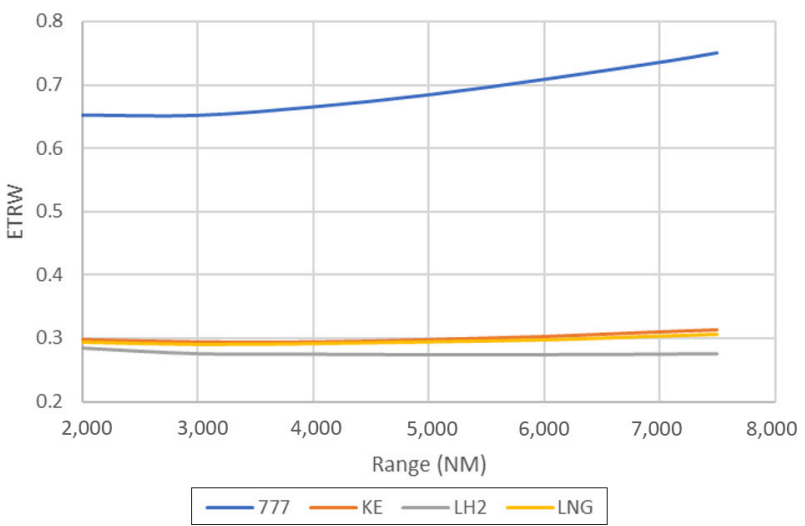


Table III Increase in energy saving potential (\%) of the N3-X variants compared to the baseline 777-200LR

\begin{tabular}{lcccccc}
\hline $\begin{array}{l}\text { Payload } \\
\text { Range }\end{array}$ & \multicolumn{3}{c}{$5,3570 \mathrm{~kg}$} & \multicolumn{4}{c}{$44,770 \mathrm{~kg}$} \\
& Kerosene & LNG & LH $_{2}$ & Kerosene & LNG & LH $_{2}$ \\
\hline $\mathbf{2 , 0 0 0}$ & 56.7 & 57.4 & 58.2 & 56.8 & 58.2 & 59.4 \\
$\mathbf{3 , 0 0 0}$ & 57.1 & 57.9 & 59.1 & 57.3 & 58.5 & 60.1 \\
$\mathbf{4 , 0 0 0}$ & 57.8 & 58.7 & 60.5 & 57.9 & 59.1 & 61.0 \\
$\mathbf{5 , 0 0 0}$ & 58.7 & 59.4 & 61.4 & 58.9 & 59.9 & 62.1 \\
$\mathbf{6 , 0 0 0}$ & 59.4 & 60.3 & 62.7 & 59.4 & 60.6 & 63.2 \\
$\mathbf{7 , 0 0 0}$ & 60.1 & 61.3 & 64.0 & 60.3 & 61.6 & 64.5 \\
$\mathbf{7 , 5 0 0}$ & 60.5 & 61.8 & 64.7 & 60.7 & 62.0 & 65.0 \\
\hline
\end{tabular}

significant benefits. According to NASA's $\mathrm{N}+3$ goals, a target of more than 60 per cent reduction in aircraft energy consumption is targeted by 2035 relative to the 2005 "best in class". In the assessment performed, it was found that the kerosene variant of the N3-X could achieve this reduction above a range of 7,000 NM, the LNG variant above a range of 6,000 NM and the $\mathrm{LH}_{2}$ variant reaches this target above a range of 5,000 NM. With a decrease in payload, this benefit is further improved. The results are summarised in the Table III.

\section{Conclusion}

The BWB with turbo-electric distributed propulsion offers advantages over current military aircraft in terms of its energy efficiency, low noise and low emissions. Additionally, when combined with fuels such as $\mathrm{LH}_{2}$ and LNG, a further decrease in fuel consumption can be obtained, and due to the reduced carbon to hydrogen ratio, $\mathrm{CO} 2$ emissions can be further reduced or eliminated. Although the assessed hydrogen-fuelled variant of the N3-X has a shorter range than the others, it could be a competitive alternative to the A330 MRTT or Boeing KC$46 \mathrm{~A}$ as it can potentially carry $180-340$ per cent more payload over 3,000 NM. There is also the potential to increase the range of the hydrogen-fuelled N3-X derivative by stretching its airframe to increase its internal volume to accommodate larger hydrogen tanks. Alternatively, the LNG fuelled variant would provide a low emissions alternative to current military aircraft and additional payload and range improvements (70-170 per cent more payload over 7,500 NM). This could allow for enhanced aerial-refuelling capabilities, as the aircraft could be kept on station for longer or used to refuel a larger number of aircraft. Although this proposal is still at the concept design stage, ongoing research could pave the way towards more energy-efficient and lower-emissions military aircraft offering operational improvements.

\section{References}

Brewer, G.D. (1976), “Aviation usage of liquid hydrogen fuelprospects and problems", International fournal of Hydrogen Energy, Vol. 1 No. 1, pp. 65-88, available at: https://doi.org/ 10.1016/0360-3199(76)90011-2 (accessed 13 September 2018).

CBO (2016), "Department of the Air Force", available at: www.cbo.gov/publication/51535 (accessed 5 August 2018).
Charpentreau, C. (2019), "The US Air Force takes delivery of long awaited Boeing KC-46A", Aerotime News Hub, available from: www.aerotime.aero/clement.charpentreau/ 22241-the-us-air-force-takes-delivery-of-long-awaited-boeingkc-46a (accessed 15 July 2018).

ESDU (1992), Acceleration factors for climb and descent rates at constant EAS, CAS, M, Data Item 81046, ESDU, London.

European Commission (2017), "Reducing emissions from aviation”, available from: https://ec.europa.eu/clima/policies/ transport/aviation (accessed 15 July 2018).

European Commission (2018), "Enabling cryogenic hydrogen based CO2 free air transport (ENABLEH2)", available at: https://trimis.ec.europa.eu/project/enabling-cryogenic-hydrogenbased-co2-free-air-transport-enableh2 (accessed 15 July 2018).

FAS (2000), “C-17 Globemaster III", available at: https://fas. org/man/dod-101/sys/ac/c-17.htm (accessed 27 August 2018).

Felder, J.L., Kim, H.D. and Brown, G.V. (2009), "Turboelectric distributed propulsion engine cycle analysis for Hybrid-wing-body aircraft", 47th AIAA Aerospace Sciences Meeting Including The New Horizons Forum and Aerospace Exposition, Orlando, FL, 5-8 January 2009, NASA Glenn Research Center, Cleveland, $\mathrm{OH}$, available at: doi. org/10.2514/6.2009-1132 (accessed 16 August 2018).

Felder, J.L., Brown, G.V., Kim, H.D. and Chu, J. (2011), "Turboelectric distributed propulsion in a hybrid wing body aircraft", 20th International Society for Airbreathing Engineers (ISABE 2011). Gothenburg, 12-16 September 2011, NASA Glenn Research Center, Cleveland, OH, pp. 1-20, available at: https://ntrs.nasa.gov/search.jsp?R=20120000856 (accessed 5 October 2018).

Funk, J.E., Harber, J.R. and Morin, L. (2006), "Future military common aircraft development opportunities", 44th AIAA Aerospace Sciences Meeting and Exhibit, Reno, NV, 9-12 January 2006, Aeronautical Systems Center, OH, available at: https://doi.org/10.2514/6.2006-1514 (accessed 18 August 2018).

GlobalSecurity (2011), "C-130H Hercules specifications", available at: www.globalsecurity.org/military/systems/aircraft/c130-specs.htm (accessed 23 August 2018).

GlobalSecurity (2015), "Airbus A330 Multi Role Tanker Transport (MRTT)", available at: www.globalsecurity.org/ military/world/europe/a330-mrtt.htm (accessed 23 August 2018).

Goldberg, C. (2018), "Techno-economic, environment and risk analysis of an aircraft concept with turbo-electric distributed propulsion", $\mathrm{PhD}$ thesis, Cranfield University, available at: https://dspace.lib.cranfield.ac.uk/handle/1826/ 3748 (accessed 20 September 2018).

IATA (2017), “2036 Forecast reveals air passengers will nearly double to 7.8 billion", available at: www.iata.org/pressroom/ pr/Pages/2017-10-24-01.aspx (accessed 2 July 2018).

Jenkinson, L.R., Simpkin, P. and Rhodes, D. (1999), Civil fet Aircraft Design, American Institute of Aeronautics and Astronautics.

Liebeck, R.H. (2004), "Design of the blended wing body subsonic transport", Fournal of Aircraft, Vol. 41 No. 1, pp. 10-25, available at: https://arc.aiaa.org/doi/abs/10.2514/ 1.9084? journalCode=ja (accessed 22 August 2018). 
MacMillan, W.L. (1974), "Development of a modular type computer program for the calculation of gas turbine off design performance", $\mathrm{PhD}$ thesis, Cranfield University, available at: www.researchgate.net/publication/35479284 Development_of_a_modular_type_computer_program_for_ the_calculation_of_gas_turbine_off_design_performance (accessed 25 September 2018).

Mari, P. (2015), "Techno-economic and environmental risk assessment of a blended wing body with distributed propulsion - liquid hydrogen variant", Master's thesis, Cranfield University, available at: http://cclibweb-3.central. cranfield.ac.uk/handle/1826.1/10798 (accessed 10 December 2018).

Moore, M. (2014), "OpenVSP model: NASA N3-X concept", available at: http://hangar.openvsp.org/vspfiles/59 (accessed 6 October 2018).

Ogaji, S., Pilidis, P. and Hales, R. (2007), TERA - a Tool for Aero-Engine Modelling and Management, Cranfield University, Cranfield, available at: www.researchgate.net/ publication/228793159_TERA-A_Tool_for_Aero-engine_ Modelling_and_Management (accessed 15 September 2018).

Tirpak, A.J. (2009), "Why the 767", available at: https://web. archive.org/web/20110429021749/ or www.airforcemagazine.com/Features/modernization/-Pages/box021307 boeing.aspx (accessed 2 April 2019).

U.S. Air Force (2019), "KC-46A Pegasus", available at: www. af.mil/About-Us/Fact-Sheets/Display/Article/104537/kc-46apegasus/ (accessed 2 July 2018).

Waitz, I.A., Lukachko, S.P. and Lee, J.J. (2005), "Military aviation and the environment: historical trends and comparison to civil aviation", Fournal of Aircraft, Vol. 42 No. 2, doi: 10.2514/1.6888, available at: www.researchgate. net/publication/254793037_Military_Aviation_and_the_ Environment_Historical_Trends_and_Comparison_to_Civil_ Aviation (accessed 20 July 2018).

Whitford, R. (1999), Design of Medium Military Transport Aircraft, Cranfield University, Cranfield, available at: https:// doi.org/10.4271/1999-01-5530 (accessed 10 September 2018).

\section{Further reading}

Airbus "A400M: delivery to the point of need", available at: www.airbus.com/defence/a400m.html (accessed 23 August 2018).

Airliners "Antonov an-225 Mriya", available at: www.airliners. net/aircraft-data/antonov-an-225-mriya/389. (accessed 23 August 2018).

National Aeronautics and Space Administration (2017), "New Aviation horizons initiative and complementary investments", available at: www.nasa.gov/sites/default/files/ atoms/files/nasa-aero-10-yr-plan-508-reduced.pdf (accessed 6 October 2018).

NACRE (2011), Final Activity Report. 2005-2010, Technical report, Sixth Framewirk programme Priority 4 Aeronautics and Space, (accessed 6 October 2018).

\section{Corresponding author}

Sajal Kissoon can be contacted at: sajalkissoon@gmail.com 
2020-04-06

\section{Assessment of the BWB aircraft for military transport}

Kissoon, Sajal

Emerald

Kissoon S, Mastropierro FS, Nalianda DK, et al (2020) Assessment of the BWB aircraft for military transport. Aircraft Engineering and Aerospace Technology, Volume 92, Issue 5, May pÿ2020, pp. 769776

https://doi.org/10.1108/AEAT-09-2019-0188

Downloaded from Cranfield Library Services E-Repository 